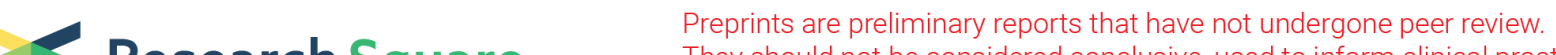 $\begin{array}{ll}\text { Research Square } & \text { They should not be considered conclusive, used to inform clinical practice, } \\ \text { or referenced by the media as validated information. }\end{array}$
}

\section{Epidemiology and clinical significance of pLVPK-like virulence plasmid in KPC-2-producing Klebsiella pneumoniae infections in eastern China: a preliminary exploration}

\section{Min Xu}

Zhejiang University School of Medicine First Affiliated Hospital

\section{Qing Yang}

Zhejiang University School of Medicine First Affiliated Hospital

\section{Yanchao Liu}

Zhejiang University School of Medicine First Affiliated Hospital

\section{Xiao Chen}

Zhejiang University School of Medicine First Affiliated Hospital Haishen Kong

Zhejiang University School of Medicine First Affiliated Hospital

\section{Zhangrong Fu}

Zhejiang University School of Medicine First Affiliated Hospital

\section{Yu Chen}

Zhejiang University School of Medicine First Affiliated Hospital

\section{Yiqi Fu ( $\square$ fuyiqi@zju.edu.cn )}

Zhejiang University School of Medicine First Affiliated Hospital

\section{Research}

Keywords: pLVPK, virulence plasmid, KPC-kp, prevalence, clinical outcome

Posted Date: August 4th, 2020

DOI: https://doi.org/10.21203/rs.3.rs-52971/v1

License: (c) (i) This work is licensed under a Creative Commons Attribution 4.0 International License. Read Full License 


\section{Abstract}

Background: By acquiring a pLVPK-like virulence plasmid (pVir), Klebsiella pneumoniae carbapenemase (KPC)-producing K. pneumoniae (KPC-kp) evolves to a hypervirulent variant, with increasing cases reported worldwide. However, little is known about the epidemiological trend of pVir in KPC-kp, as well as clinical characteristics of infections caused by this novel strain ( $\left.p V i r^{+}-K P C-k p\right)$.

Methods: From 2014-2018, 662 carbapenem-resistant K. pneumoniae (CRKP) were consecutively collected from a tertiary hospital of eastern China. The confirmed KPC-kp were subjected to antimicrobial susceptibility testing, multilocus sequence typing and detection of pLVPK-related genetic loci ( $r m p A$, rmpA2, iucA and iroN) to identify $\mathrm{pVir}{ }^{+}-\mathrm{KPC}-\mathrm{kp}$ strains. Then, the clinical characteristics and outcomes of $\mathrm{KPC}-\mathrm{kp}$ infection patients with and without $\mathrm{pVir}$ were compared. Moreover, the risk factors for $\mathrm{pVir}{ }^{+}-\mathrm{KPC}-$ $\mathrm{kp}$ infections also determined by a multivariable logistic regression analysis.

Results: of the 662 CRKP, 86.6\% (573/662) were KPC-kp including 285 (49.7\%) pVir-KPC-kp and 288 (50.3\%) pVir-KPC-kp. Notably, the prevalence of pVir ${ }^{+}-K P C-k p$ by year increased remarkably from 2014 $(19.5 \%, 8 / 41)$ to $2018(60.0 \%, 90 / 150)$. Sequence type (ST) 11 was the most predominant ST, accounting for $88.9 \%$ of all pVir ${ }^{+}-K P C-k p$. For the 352 KPC-kp infection cases (186 with pVir ${ }^{+}-K P C-k p$ and 166 with pVir-KPC-kp), multivariable analysis indicated neurosurgery (Odds ratio [OR], 2.92; 95\% confidence interval [Cl], 1.48-5.75; $P=0.002$ ) was independently associated with $\mathrm{pVir}{ }^{+}-\mathrm{KPC}-\mathrm{kp}$ infections. Although patients with pVir ${ }^{+}-\mathrm{KPC}-\mathrm{kp}$ infections had higher incidence of septic shock (31.2\% vs. $\left.21.1 \%, P=0.03\right)$, the two groups showed no significant differences in 7-day mortality ( $23.1 \%$ vs. $18.1 \%, P=0.24)$ or 28 -day mortality $(45.7 \%$ vs. $44.0 \%, P=0.75)$.

Conclusions: Altogether, wide dissemination of pVir in ST11 KPC-kp has emerged in China. Neurosurgery is an independent risk factor for acquisition of $\mathrm{pVir}^{+}-\mathrm{KPC}-\mathrm{kp}$ infections. The mortality rates were similar between patients infected with $p V i r^{+}-K P C-k p$ or $p V i r^{-}-K P C-k p$, suggesting uncertain impact of $p V i r$ in clinical outcome.

\section{Background}

During the last decades, a new variant called hypervirulent Klebsiella pneumoniae (hvKP) causing severe invasive infections in apparently healthy people has posed a particular threat to public health $[1,2]$. pLVPK originates from K. pneumoniae CG43 strain [3]. Virulence plasmid carrying pLVPK-borne genes termed as pLVPK-like plasmid ( $\mathrm{pVir}$ ) is commonly detected in hvKP clones, such as sequence type (ST) 23 [4]. A correlation has been established between pVir and high-level virulence [5]. Some virulence genes harbored by $\mathrm{pVir}$, including $r m p A / r m p A 2$ (regulating capsular polysaccharide biosynthesis), iucABCD-iutA (encoding aerobactin siderophore) and $\operatorname{irOBCDN}$ (encoding salmochelin siderophore), are proved to be better biomarkers for hvKP than the widely used "string test" $[1,6]$. 
Contrary to hvKP, carbapenem-resistant $K$. pneumoniae (CRKP) is recognized as an important pathogen associated with nosocomial infections in immunocompromised patients [7]. Since first reported in China in 2007 [8], Klebsiella pneumoniae carbapenemase (KPC) has become the most predominant contributor to carbapenem resistance in CRKP of China [9]. It is a common sense that KPC-producing K. pneumoniae (KPC-kp) is absent of pVir and nearly avirulent experimentally [10]. However, convergent $K$. pneumoniae strains of widely distributed ST11 carrying both pVir and KPC (pVir $\left.{ }^{+}-K P C-k p\right)$ have emerged, especially in China [11-13]. So far, most cases of pVir ${ }^{+}-K P C-k p$ infections were still isolated or sporadic. However, recently, new evidence alerted that a rapidly increasing prevalence of $\mathrm{pVir}{ }^{+}-\mathrm{KPC}-\mathrm{kp}$ might occur due to the potential spread of a novel conjugative IncFIB plasmid containing $b / a_{K P C}$ and key virulence-encoding region of pVir simultaneously $[14,15]$. Therefore, there is an urgent need to evaluate the current epidemiological trend of pVir in KPC-kp.

Besides displaying high levels of resistance to commonly used antibiotics, $\mathrm{pVir}{ }^{+}-\mathrm{KPC}-\mathrm{kp}$ strains in general exhibited enhanced anti-phagocytic and serum resistance properties in vitro and were associated with extremely high mortality both in affected patients and experimental models [11, 12]. However, the association of virulence traits and $\mathrm{pVir}$ in multidrug-resistant $K$. pneumoniae remains controversial and the clinical features of patients infected with pVir ${ }^{+}-K P C-k p$ strains are less revealed $[16,17]$.

To address these problems, in present study, we first investigated the prevalence of pVir among KPC-kp collected from a tertiary hospital in eastern China over four consecutive years. Then, we characterized the clinical features and outcomes of $\mathrm{pVir}{ }^{+}-\mathrm{KPC}-\mathrm{kp}$ infections by comparing with $\mathrm{pVir}-\mathrm{KPC}-\mathrm{kp}$ strains. Also, the risk factors of $\mathrm{pVir}{ }^{+}-\mathrm{KPC}-\mathrm{kp}$ infections were determined.

\section{Methods}

\section{Design, Setting and Ethics}

This retrospective cohort study was performed in The First Affiliated Hospital, College of Medicine, Zhejiang University, from July 2014 to August 2018. It is one of the largest hospitals in eastern China, having approximate 2500 beds and 131,000 annual admissions. The first report of KPC-kp in China came from here in 2007 [8]. Since then, KPC-kp has been endemic in the hospital [18].

The present study was conducted according to the principles stated in the Declaration of Helsinki and approved by the ethical review board of The First Affiliated Hospital, College of Medicine, Zhejiang University.

\section{Patient Cohort}

The source population consisted of all patients admitted to the hospital during the study period, whose bacterial cultures were identified as CRKP. The clinical specimens mainly included blood, sputum/tracheal aspirate, pleural fluid and ascites. Further detailed collection of clinical information was performed for the patients whose isolates were verified as KPC-kp. Patients aged $<18$ years and those 
with missing or incomplete clinical records were excluded. Patients with polymicrobial infections and those with KPC-kp colonization were excluded as well. Infection or colonization was defined according to the criteria of the Center for Disease Control and Prevention of America [19]. Only the first episode for each patient was included in our analysis.

\section{Clinical Data Collection}

For all eligible KPC-kp infection cases, chart review was performed to obtain the clinical data. Infectious disease specialists independently collected the following clinical information using the patients' electronic charts and/or paper records: demographic conditions, unit and duration of hospitalization, underlying diseases, Charlson Comorbidity Index, abscess information and previous invasive procedures. Patients were defined as immunosuppressed if they had HIV or AIDS, were post-transplant, had received chemotherapy within 4 weeks, had received immunosuppressive agents for more than 2 weeks, or had neutropenia. Previous use of antibiotics was defined as antimicrobial therapy (intravenous or oral) within 4 weeks before infections. Invasive procedures included surgery and mechanical ventilation performed within 4 weeks prior to the infections. Appropriate empirical or definitive antimicrobial therapy was defined as previously described [18].

\section{Outcome Variables}

The primary outcome variables were all-cause mortality at day 7 and day 28. Other secondary outcome variables were infection-related mortality and in-hospital mortality. Septic shock and multiple organ dysfunction syndrome (MODS) at the onset of infections, as well as the length of stay (LOS) from culture to discharge, were also assessed.

\section{Bacterial Identification and Antimicrobial Susceptibility Testing}

Bacterial isolates identification and antimicrobial susceptibility testing were performed using Vitek 2 automated system (bioMérieux, France) and the results were interpreted according to Clinical and Laboratory Standards Institute (CLSI) 2019 criteria [20]. For tigecycline, colistin and ceftazidimeavibactam (CAZ/AVI), minimum inhibitory concentrations (MICs) were determined using broth microdilution method. The susceptibility results were categorized in accordance with the CLSI criteria for CAZ/AVI, while European Committee on Antimicrobial Susceptibility Testing (version 9.0, http://www.eucast.org/clinical_breakpoints/) for colistin and tigecycline. The species identification was verified by MALDI-TOF MS (Bruker Daltonics Inc., Fremont, CA, USA). Pseudomonas aeruginosa ATCC27853 and Escherichia coli ATCC 25922 were used as the quality control strains of antimicrobial susceptibility testing.

\section{Molecular Analysis}

All of the CRKP were subjected to detection of $b / a_{\mathrm{KPC}}$ by PCR. The isolates carrying $b / a_{\mathrm{KPC}}$ were further investigated for pLVPK-related genetic loci $r m p A, r m p A 2$, iucA and iroN. The primers used for PCR 
amplification and sequencing were acquired from a previous study [11]. Considering the primers of $r m p A$ and $r m p A 2$ target only the plasmid-borne genes, while iucA and iroN for both plasmid-borne and chromosomally-encoded genes, KPC-kp isolates of positive $r m p A$ or $r m p A 2$ were defined as $\mathrm{pVir}{ }^{+}-\mathrm{KPC}-\mathrm{kp}$ according to the definition previously described [11, 21-23].

Multilocus sequence typing (MLST) was carried out according to the protocol described on the $K$. pneumoniae MLST website ((https://bigsdb.pasteur.fr/klebsiella/klebsiella.html), and the results were analysed using the corresponding database.

\section{Statistical Analysis}

Data were analysed using SPSS software (version 20; SPSS Inc., Chicago, IL, USA). Categorical variables were presented as absolute numbers and their relative frequencies, and analyzed by chi-square or Fisher exact test. For continuous variables, the Kolmogorov-Smirnov test was applied to distinguish between normal and non-normal distribution. Normally distributed data were expressed as mean and standard deviation (SD) and analysed using Student's t-test, while non-normally distributed data were present with median and interquartile rang (IQR) and compared using Mann-Whitney test. To identify independent predictors for $\mathrm{pVir}{ }^{+}-\mathrm{KPC}-\mathrm{kp}$ infections, we used logistic regression. All variables with $P$ value $<0.1$ in univariate analysis as well as clinical importance were incorporated into multivariable regression model. $P$ value $<0.05$ was considered statistically significant.

\section{Results}

During the study period, a total of 662 CRKP isolates were collected. Of these, 573 were KPC-kp, and further were analysed for antimicrobial testing and pLVPK-derived loci. Two hundred and eighty-five isolates were identified as pVir ${ }^{+}-\mathrm{KPC}-\mathrm{kp}$ and 288 as pVir-KPC-kp. There were 352 cases met the criteria and included in the final analysis of clinical characteristics. The causes of exclusion are shown in Figure 1 , mainly polymicrobial infections (104 cases) and colonization (86 cases).

\section{Prevalence of pVir in KPC-kp}

The proportion of pVir among KPC-kp isolates increased continuously and significantly between 2014 and 2018 (19.5\% in 2014, 18.5\% in 2015, 49.5\% in 2016, 77.5\% in 2017 and 60.0\% in 2018, respectively). Among the $285 \mathrm{pVir}+$-KPC-kp isolates, pVir $\left(r m p A^{+} r m p A 2^{+} i u c A^{+} i r o N^{+}\right)$and pVir $\left(r m p A 2^{+} i u c A^{+}\right)$were the two most predominant virulence plasmid types, accounting for $55.1 \%(157 / 285)$ and $28.4 \%(81 / 285)$ respectively. The annual distribution of different types of pVir was shown in Figure 2 and Table 51.

Among the $285 \mathrm{pVir}{ }^{+}-\mathrm{KPC}-\mathrm{kp}, 90$ isolates were randomly selected for MLST analysis. Overall, these strains were assigned to seven different STs, with ST11 the most common type (88.9\%, 80/90), followed by ST23 $(3.3 \%, 3 / 90)$ and ST1660 $(2.2 \%, 2 / 90)$. Additionally, ST65, ST152, ST660, ST893 and ST268 were found in one isolate each. 


\section{Antimicrobial resistance}

Susceptibility testing showed significantly higher resistance rates of aminoglycosides, cefepime and trimethoprim-sulfamethoxazole in pVir ${ }^{+}-\mathrm{KPC}-\mathrm{kp}$ than pVir-KPC-kp $(P<0.001,=0.02$ and $<0.001$, respectively). However, the CZA/AVI resistance rate of pVir-KPC-kp was $4.9 \%$, which was much higher than that of $\mathrm{pVir}{ }^{+}-\mathrm{KPC}-\mathrm{kp}(4.9 \%$ vs. $0.35 \%, P=0.001)$. Although the results did not reach statistical significance, the resistance rates of $\mathrm{pVir}{ }^{+}-\mathrm{KPC}-\mathrm{kp}$ against tigecycline and colistin were higher than those of pVir-KPC-kp (Figure 3, Table S2).

\section{Predictors and Survival Impact of pVir-KPC-kp Infections}

The clinical characteristics of the cohort are described in Table 1. Among the 352 episodes of KPC-kp infections, 186 (52.8\%) were due to pVir ${ }^{+}-K P C-k p$ and 166 (47.2\%) were due to pVir-KPC-kp. The age, sex, hospital ward and abscess formation did not differ between these two groups. The source of infections and prior antibiotic exposure except cephalosporins (pVir ${ }^{+}-\mathrm{KPC}-\mathrm{kp} 7.0 \%$ vs. pVir-KPC-kp 2.4\%, $P=0.045$ ) in both groups were similar as well. pVir-KPC-kp infections were more associated with immunosuppression state (35.5\% vs. $25.3 \%, P=0.04)$. In contrast, a significantly more patients with $\mathrm{pVir}{ }^{+}-$ KPC-kp infections had Charlson comorbidity index $<3(36.6 \%$ vs. $23.5 \%, P=0.008)$. The pVir ${ }^{+}-\mathrm{KPC}-\mathrm{kp}$ infections were also more associated with underlying cerebral vascular disease $(25.8 \%$ vs. $15.7 \%, P$ $=0.02)$ and performance of neurosurgery within 4 weeks prior to infections $(22.0 \%$ vs. $7.8 \%, P<0.001)$ (Table 1). However, only neurosurgery remained associated with $\mathrm{pVir}{ }^{+}-\mathrm{KPC}-\mathrm{kp}$ infections in multivariable analysis (OR, 2.92; 95\% Cl, 1.48-5.75; $P=0.002$ ) (Table 2).

Unexpectedly, although patients infected with $\mathrm{pVir}{ }^{+}-\mathrm{KPC}-\mathrm{kp}$ had higher incidence of septic shock (31.2\% vs. $21.1 \%, p=0.03$ ), the two groups did not differ significantly with respect to 7 -day, 28-day, overall and infected-related mortality (Table 3 ).

Furthermore, multivariate analysis of 130 patients with bloodstream infections and 126 intensive care unit (ICU) patients also indicated neurosurgery was the only independent predictor for $\mathrm{pVir}{ }^{+}-\mathrm{KPC}-\mathrm{kp}$ infections. Besides, among these patients, those infected with $p V i r^{+}-K P C-k p$ and pVir-KPC-kp had similar clinical outcomes as well (data not shown).

\section{Discussion}

The present study found a high prevalence and increasing trend of pVir in ST11 KPC-kp in our hospital during the past four years. We identified previous neurosurgery within 4 weeks as the only clinical factor independently associated with pVir ${ }^{+}-K P C-k p$ infections. Although septic shock was more frequently occurred in patients with $\mathrm{pVir}{ }^{+}-\mathrm{KPC}-\mathrm{kp}$, the two groups did not differ significantly in terms of mortality. Most recently, a multicenter molecular epidemiological study of carbapenem-resistant hypervirulent $K$. 
pneumoniae (CR-hvKP) in China was reported, while limited clinical data was available [24]. As our best knowledge, it is so far the largest retrospective cohort study of KPC-kp with and without pVir.

Firstly, we screened the carriage of pVir as previously described [11]. Consistent with previous reports, most pVir'-KPC-kp belonged to the epidemic ST11 $[23,25,26]$. Notably, the prevalence of pVir in KPC-kp in 2014 had already reached 19.5\%, much higher than that reported from Gu et al (3\% in 2015) [11], suggesting an earlier transmission timeline in our hospital. Most recently, the research of Yang et al indicated a $100 \mathrm{~kb}$ fragment of $\mathrm{pVir}$ integrated into a transmissible b/a $a_{\mathrm{KPC}}$-bearing IncFIB plasmid, which would prompt the dissemination of pVir among CRKP [14]. Based on the facts that the detection of $\mathrm{pVir}^{+}-$ KPC-kp increased from 2014 to 2018 and ST11 pVir ${ }^{+}-K P C-k p$ gradually becomes to be the predominant clone, it is speculated a transmission of IncFIB plasmid carrying both pVir-fragment and $b / a_{\mathrm{KPC}}$ among ST11 KPC-kp might have happened in our region. Studies aiming to test this hypothesis would be very imperative. Furthermore, we found nearly half of the pVir ${ }^{+}-\mathrm{KPC}-\mathrm{kp}(44.9 \%)$ were absent of one or more pVir-featured genes, suggesting common deletion events in the process of plasmid fragment integration.

As most studies focused on the molecular epidemiological investigation of pVir ${ }^{+}-\mathrm{KPC}-\mathrm{kp}$ [24, 25], little was known about the risk factors of infections caused by this newly emerged variant. Here, we showed that previous neurosurgery within 4 weeks could help clinicians to identify patients of high risk to acquire pVir ${ }^{+}-$KPC-kp infections $(p=0.002)$. Our previous study reported that about half of $K$. pneumoniae meningitis was caused by pVir'-KPC-kp in our hospital from 2011 to 2017 [27]. In order to establish the correlation of neurosurgery and meningitis, we performed subgroup analysis of post-neurosurgery patients. However, it is of note that, most of the post-neurosurgical patients $(46.3 \%, 25 / 54)$ were presence of pVir ${ }^{+}-\mathrm{KPC}-\mathrm{kp}$ pneumonia and $35.1 \%$ developed to bacterial meningitis. There was no statistical significance of detection sites of pVir ${ }^{+}-\mathrm{KPC}-\mathrm{kp}$ between post-neurosurgical patients and other patients. Moreover, for the patients of bloodstream infection and patients in ICU, neurosurgery within 4 weeks was also the independent factors of $\mathrm{pVir}{ }^{+}-\mathrm{KPC}-\mathrm{kp}$ infections. Further prospective survey and molecular epidemiology will clarify the correlation between neurosurgery and pVir ${ }^{+}-\mathrm{KPC}-\mathrm{kp}$ infection, as well as the mode of transmission.

In K. pneumoniae, the terms "hypervirulence" primarily referred to isolates displaying hypermucoviscosity and causing invasive infections. However, most recent studies viewed the $\mathrm{pVir}{ }^{+}-\mathrm{KPC}-\mathrm{kp}$ strains associated with an extremely high infectious mortality as CR-hvKP, even if there was a lack of clinical features due to hvKP infections [11, 13]. In present study, we compared some well-recognized parameters that could assist in differentiating infection due to hypervirulent and classical $K$. pneumoniae strains (such as infection development location, patients' age and abscess formation) [1], there was no difference between two groups. Moreover, our results indicated the 7-day, 28-day, overall and infection-related mortality were similar between patients with $\mathrm{pVir}{ }^{+}-\mathrm{KPC}-\mathrm{kp}$ and pVir-KPC-kp infections. Although the inconsistencies in patient care or variability in types of infections might introduce bias to the analysis, further evaluation of the association between $\mathrm{pVir}{ }^{+}-\mathrm{KPC}-\mathrm{kp}$ infections and mortality for ICU patients and patients with BSIs drew the same conclusion. Furthermore, we also noted $11.6 \%$ patients $(33 / 285)$ 
isolated with $\mathrm{pVir}{ }^{+}-\mathrm{KPC}-\mathrm{kp}$ were absent of any symptoms of infections, who eventually did not develop to invasive infections and had favorable outcomes. Therefore, it was reasonable to believe not all of the KPC-kp would evolve to hypervirulent variants and resulted in fatalities by acquiring pVir. Study on the comprehensive genome analysis of ST11 pVir ${ }^{+}-\mathrm{KPC}-\mathrm{kp}$ strains with varied virulence level is underway to provide more clues on the virulence mechanisms of this newly emerged strains.

There are some important limitations of our current study that should be acknowledged. First, the study was a single-center study with KPC-kp infected patients enrolled retrospectively. Our hospital located in Zhejiang province in China, where ST11 KPC-kp was epidemic [18] and a fatal outbreak caused by ST11 CR-hvKP was reported here in 2017 [11], therefore, the generalization of our results should be approached with caution. Second, the virulence of serial pVir ${ }^{+}-\mathrm{KPC}-\mathrm{kp}$ strains that associated with variable prognosis had not be evaluated experimentally, and we just took the clinical mortality as the virulence measure. However, the present study firstly compared the clinical outcomes between patients infected with pVir ${ }^{+}-$ KPC-kp and pVir-KPC-kp.

\section{Conclusions}

Altogether, we observed a high prevalence of pVir in KPC-kp and ST11 KPC-kp with pVir as the predominant clone in our region. Moreover, we also demonstrated the clinical features and mortality was similar between infections caused by KPC-kp with and without pVir. Our finding indicated that not all of the KPC-kp could evolve to hypervirulent variant by acquiring pVir, therefore, the notion that KPC-kp harboring pVir was equivalent to hypervirulent strain should be strongly considered. Regardless, in order to prevent the dissemination of $K$ pneumoniae strain with hyper-resistance and hypervirulence, active surveillance for these KPC-kp strains carrying pVir alone with virulent evaluation was still necessary. Additionally, research into exploration of virulence mechanism of $\mathrm{pVir}^{+}-\mathrm{KPC}-\mathrm{kp}$ strains may help to treat these infections.

\section{Abbreviations}

pVir: pLVPK-like virulence plasmid, KPC: Klebsiella pneumoniae carbapenemases, KPC-kp: (KPC)producing K. pneumoniae, CRKP, Carbapenem-resistant K. pneumoniae, ST: Sequence type, OR: Odds ratio, Cl: Confidence interval, hvKP: Hypervirulent Klebsiella pneumoniae, MODS: Multiple organ dysfunction syndrome, LOS: Length of stay, CLSI: Clinical and Laboratory Standards Institute, CAZ/AVI: Ceftazidime-avibactam, MICs: Minimum inhibitory concentrations, MLST ; Multilocus sequence typing, SD: Standard deviation, ICU, intensive care unit, CR-hvKP: Carbapenem-resistant hypervirulent $K$. pneumoniae.

\section{Declarations}

\section{Ethics approval and consent to participate}


Ethical approval was granted from the Ethics Committees and review board of the First Affiliated Hospital, College of Medicine, Zhejiang University. As this study used secondary data, informed consent was not obtained from patients.

\section{Consent for publication}

Not applicable.

\section{Availability of data and materials}

The data that support the findings of this study are available from the corresponding author on reasonable request.

\section{Competing interests}

The authors declare that they have no competing interests.

\section{Funding}

This work was partially supported by the National Natural Science Foundation (no 81301459), Natural Science Foundation of Zhejiang Province (no LQ13H186001), and National Key Program for Infectious Diseases of China (no2017ZX10103008).

\section{Authors' contributions}

MX and YF designed the study. MX and QY performed data analysis and drafted the manuscript. YL performed the genotypic analysis of the clinical isolates. HK and ZF participated in the collection of strains and clinical information. XC performed the antimicrobial susceptibility testing. YC and YF reviewed the paper and provided recommendations. All authors read and approved the final manuscript.

\section{Acknowledgments}

We thank the entire staff at the Department of Microbiology, The First Affiliated Hospital, College of Medicine, Zhejiang University, for their daily contributions to this study. We also thank the team of curators from the Institute Pasteur MLST (Paris, France) for importing novel alleles and profiles at http://bigsdb.pasteur.fr/klebsiella/klebsiella.html.

\section{Reference}

1. Russo TA, Marr CM. Hypervirulent Klebsiella pneumoniae. Clin Microbiol Rev. 2019;32.

2. Paczosa MK, Mecsas J. Klebsiella pneumoniae. Going on the offense with a strong defense. Microbiol Mol Biol Rev. 2016;80:629-61.

3. Chen YT, Chang HY, Lai YC, Pan CC, Tsai SF, Peng HL. Sequencing and analysis of the large virulence plasmid pLVPK of Klebsiella pneumoniae CG43. Gene. 2004;337:189-98. 
4. Yu F, Lv J, Niu S, Du H, Tang YW, Pitout JDD, et al. Multiplex PCR analysis for rapid detection of Klebsiella pneumoniae carbapenem-resistant (sequence type 258 [ST258] and ST11) and hypervirulent (ST23, ST65, ST86, and ST375) strains. J Clin Microbiol. 2018;56.

5. Tang HL, Chiang MK, Liou WJ, Chen YT, Peng HL, Chiou CS, et al. Correlation between Klebsiella pneumoniae carrying pLVPK-derived loci and abscess formation. Eur J Clin Microbiol Infect Dis. 2010;29:689-98.

6. Russo TA, Olson R, Fang CT, Stoesser N, Miller M, MacDonald U, et al. Identification of biomarkers for differentiation of hypervirulent Klebsiella pneumoniae from classical K. pneumoniae. J Clin Microbiol. 2018;56.

7. Lee CR, Lee JH, Park KS, Kim YB, Jeong BC, Lee SH. Global dissemination of carbapenemaseproducing Klebsiella pneumoniae: epidemiology, genetic context, treatment options, and detection methods. Front Microbiol. 2016;7:895.

8. Wei ZQ, Du XX, Yu YS, Shen P, Chen YG, Li LJ. Plasmid-mediated KPC-2 in a Klebsiella pneumoniae isolate from China. Antimicrob Agents Chemother. 2007;51:763-5.

9. Zhang R, Liu L, Zhou H, Chan EW, Li J, Fang Y, et al. Nationwide surveillance of clinical carbapenemresistant enterobacteriaceae (CRE) strains in China. EBioMedicine. 2017;19:98-106.

10. Ramirez MS, Traglia GM, Lin DL, Tran T, Tolmasky ME. Plasmid-mediated antibiotic resistance and virulence in gram-negatives: the Klebsiella pneumoniae paradigm. Microbiol Spectr. 2014;2.

11. Gu D, Dong N, Zheng Z, Lin D, Huang M, Wang L, et al. A fatal outbreak of ST11 carbapenemresistant hypervirulent Klebsiella pneumoniae in a Chinese hospital: a molecular epidemiological study. Lancet Infect Dis. 2018;18:37-46.

12. Huang $\mathrm{YH}$, Chou SH, Liang SW, Ni CE, Lin YT, Huang YW, et al. Emergence of an XDR and carbapenemase-producing hypervirulent Klebsiella pneumoniae strain in Taiwan. J Antimicrob Chemother. 2018;73:2039-46.

13. Zhao Y, Zhang X, Torres VVL, Liu H, Rocker A, Zhang Y, et al. An Outbreak of carbapenem-resistant and hypervirulent Klebsiella pneumoniae in an intensive care unit of a major teaching hospital in Wenzhou, China. Front Public Health. 2019;7:229.

14. Yang $X$, Wai-Chi Chan $E$, Zhang $R$, Chen $S$. A conjugative plasmid that augments virulence in Klebsiella pneumoniae. Nat Microbiol. 2019;4:2039-43.

15. Yang $X, Y e L, C h a n E W$, Zhang $R$, Chen $S$. Tracking recombination events that occur in conjugative virulence plasmid p15WZ-82_Vir during the transmission process. mSystems. 2020;5.

16. Shu L, Dong N, Lu J, Zheng Z, Hu J, Zeng W, et al. Emergence of OXA-232 carbapenemase-producing Klebsiella pneumoniae that carries a pLVPK-Like virulence plasmid among elderly patients in China. Antimicrob Agents Chemother. 2019;63.

17. Fu Y, Xu M, Liu Y, Li A, Zhou J. Virulence and genomic features of a bla $a_{\mathrm{CTX}-\mathrm{M}-3}$ and $b / a_{\mathrm{CTX}-\mathrm{M}-14}$ coharboring hypermucoviscous Klebsiella pneumoniae of serotype K2 and ST65. Infect Drug Resist. 2019;12:145-59. 
18. Xu M, Fu Y, Kong H, Chen X, Chen Y, Li L, et al. Bloodstream infections caused by Klebsiella pneumoniae: prevalence of $b / a_{\mathrm{KPC}}$, virulence factors and their impacts on clinical outcome. BMC Infect Dis. 2018;18:358.

19. Centers for Disease Control and Prevention. CDC/NHSN Surveillance Definitions of Specific Types of Infections. Atlanta: CDC. 2017.

20. Clinical and Laboratory Standards Institute. Performance standards for antimicrobial susceptibility testing 28th informational supplement. CLSI document M100-S28. Wayne: Clinical and Laboratory Standards Institute; 2018.

21. Du P, Zhang Y, Chen C. Emergence of carbapenem-resistant hypervirulent Klebsiella pneumoniae. Lancet Infect Dis. 2018;18:23-24.

22. Wong MHY, Shum HP, Chen JHK, Man MY, Wu A, Chan EW, et al. Emergence of carbapenem-resistant hypervirulent Klebsiella pneumoniae. Lancet Infect Dis. 2018;18:24.

23. Yao H, Qin S, Chen S, Shen J, Du XD. Emergence of carbapenem-resistant hypervirulent Klebsiella pneumoniae. Lancet Infect Dis. 2018;18:25.

24. Zhang $Y$, Jin L, Ouyang P, Wang Q, Wang R, Wang J, et al. Evolution of hypervirulence in carbapenemresistant Klebsiella pneumoniae in China: a multicentre, molecular epidemiological analysis. $\mathrm{J}$ Antimicrob Chemother. 2020;75:327-36.

25. Yu F, Lv J, Niu S, Du H, Tang YW, Bonomo RA, et al. In vitro activity of ceftazidime-avibactam against carbapenem-resistant and hypervirulent Klebsiella pneumoniae isolates. Antimicrob Agents Chemother. 2018;62.

26. Zheng B, Xu H, Lv T, Guo L, Xiao Y, Huang C, et al. Stool samples of acute diarrhea inpatients as a reservoir of ST11 hypervirulent KPC-2-producing Klebsiella pneumoniae. mSystems. 2020;5.

27. Xu M, Fu Y, Fang Y, Xu H, Kong H, Liu Y, et al. High prevalence of KPC-2-producing hypervirulent Klebsiella pneumoniae causing meningitis in Eastern China. Infect Drug Resist. 2019;12:641-53.

\section{Tables}

Table 1. Clinical characteristics of 352 patients with KPC-kp infections divided into groups by presence of pVir. 


\begin{tabular}{|c|c|c|c|c|}
\hline Variable & $\begin{array}{l}\text { All isolates } \\
(n=352)\end{array}$ & $\begin{array}{l}\text { pVir }{ }^{+}-K P C-k p \\
(n=186)\end{array}$ & $\begin{array}{l}\text { pVir-KPC-kp } \\
(n=166)\end{array}$ & $\begin{array}{l}P \\
\text { value }\end{array}$ \\
\hline \multicolumn{5}{|l|}{ Demographics data } \\
\hline Age, years, (mean $\pm S D$ ) & $59.1 \pm 16.3$ & $57.9 \pm 15.7$ & $60.4 \pm 16.8$ & 0.15 \\
\hline Male gender & $248(70.5)$ & $130(69.9)$ & $118(71.1)$ & 0.81 \\
\hline $\begin{array}{l}\text { Charlson comorbidity index } \\
<3\end{array}$ & $107(30.4)$ & $68(36.6)$ & $39(23.5)$ & 0.008 \\
\hline \multicolumn{5}{|l|}{$\begin{array}{l}\text { Ward submitting index } \\
\text { culture }\end{array}$} \\
\hline ICU & $126(35.8)$ & $66(35.5)$ & $60(36.1)$ & 0.9 \\
\hline Medical & $169(48.0)$ & $86(46.2)$ & $83(50.0)$ & 0.48 \\
\hline Surgical & $55(15.6)$ & $34(18.3)$ & $21(12.7)$ & 0.15 \\
\hline \multicolumn{5}{|l|}{ Underlying diseases } \\
\hline Hypertension & $132(37.5)$ & $70(37.6)$ & $62(37.3)$ & 0.96 \\
\hline Diabetes mellitus & $74(21.0)$ & $35(18.8)$ & $39(23.5)$ & 0.29 \\
\hline Hematological malignancy & $23(6.5)$ & $9(4.8)$ & $14(8.4)$ & 0.17 \\
\hline Solid tumor & $78(22.2)$ & $37(19.9)$ & $41(24.7)$ & 0.28 \\
\hline Cirrhosis & $42(11.9)$ & $18(9.7)$ & $24(14.5 \rrbracket$ & 0.17 \\
\hline Solid organ transplantation & $60(17.0)$ & $27(14.5)$ & $33(19.9)$ & 0.18 \\
\hline Chronic kidney disease & $40(11.4)$ & $16(8.6)$ & $24(14.5)$ & 0.08 \\
\hline Chronic lung disease & $36(10.2)$ & $22(11.8)$ & $14(8.4)$ & 0.29 \\
\hline Cardiovascular disease & $63(17.9)$ & $33(17.7)$ & $30(18.1)$ & 0.94 \\
\hline Trauma & $49(13.9)$ & $30(16.1)$ & $19(11.4)$ & 0.21 \\
\hline Cerebral vascular disease & $74(21.0)$ & $48(25.8)$ & $26(15.7)$ & 0.02 \\
\hline \multicolumn{5}{|l|}{ Abscess formation } \\
\hline Liver abscess & $3(0.9)$ & $1(0.5)$ & $2(1.2)$ & 0.60 \\
\hline Non-hepatic abscess & $28(8.0)$ & $16(8.6)$ & $12(7.2)$ & 0.64 \\
\hline Non-abscess & $321(91.2)$ & $169(90.9)$ & $152(91.6)$ & 0.82 \\
\hline \multicolumn{5}{|l|}{ Invasive procedures } \\
\hline Surgery before infection & $177(50.3)$ & $102(54.8)$ & $75(45.2)$ & 0.07 \\
\hline Neurosurgery & $54(15.3)$ & $\begin{array}{l}41(22.0) \\
12 / 18\end{array}$ & $13(7.8)$ & $<0.001$ \\
\hline
\end{tabular}




\begin{tabular}{|lllll|}
\hline Mechanical ventilation & $162(46.3)$ & $91(48.9)$ & $71(43.3)$ & 0.29 \\
\hline Detection site & & & & \\
\hline Blood stream & $130(36.9)$ & $64(34.4)$ & $66(39.8)$ & 0.3 \\
\hline Low respiratory tract & $112(31.8)$ & $62(33.3)$ & $50(30.1)$ & 0.52 \\
\hline Intra-abdominal & $58(16.5)$ & $28(15.1)$ & $30(18.1)$ & 0.45 \\
\hline Cerebrospinal fluid & $23(6.5)$ & $13(7.0)$ & $10(6.0)$ & 0.72 \\
\hline Urogenital & $20(5.7)$ & $13(7.0)$ & $7(4.2)$ & 0.26 \\
\hline Skin and soft tissue & $9(2.6)$ & $6(3.2)$ & $2(1.8)$ & 0.51 \\
\hline Prior antibiotic exposure & & & & 0.045 \\
\hline Cephalosporins & $17(4.8)$ & $13(7.0)$ & $4(2.4)$ & 0.34 \\
\hline $\begin{array}{l}\text { B-lactam and } \beta \text {-lactamase } \\
\text { inhibitors }\end{array}$ & $112(31.8)$ & $55(29.6)$ & $57(34.3)$ & 0.66 \\
\hline Carbapenems & $142(40.3)$ & $73(39.2)$ & $69(41.6)$ & 1.00 \\
\hline Aminoglycosides & $2(0.6)$ & $1(0.5)$ & $1(0.6)$ & 0.86 \\
\hline Fluoroquinolones & $10(2.8)$ & $5(2.7)$ & $5(3.0)$ & 0.29 \\
\hline Tigecycline & $52(14.8)$ & $31(16.7)$ & $21(12.7)$ & 0.46 \\
\hline Others & $16(4.5)$ & $7(3.8)$ & $9(5.4)$ & $59(35.5)$ \\
\hline Immunosuppression state & $106(30.1)$ & $47(25.3)$ & & 0.04 \\
\hline
\end{tabular}

Data are presented as No. (\%) unless otherwise specified;

ICU, intensive care unit.

Table 2. Univariable and multivariable analysis of risk factors for pVir+-KPC-kp infections. 


\begin{tabular}{|c|c|c|c|c|c|c|}
\hline \multirow[t]{2}{*}{ Variable } & \multicolumn{3}{|c|}{ Univariate analysis } & \multicolumn{3}{|c|}{ Multivariate analysis } \\
\hline & OR & $95 \% \mathrm{Cl}$ & $\begin{array}{l}P \\
\text { value }\end{array}$ & OR & $\begin{array}{l}95 \% \\
\mathrm{Cl}\end{array}$ & $\begin{array}{l}P \\
\text { value }\end{array}$ \\
\hline Charlson comorbidity index $<3$ & 1.56 & $\begin{array}{l}1.12- \\
2.17\end{array}$ & 0.008 & & & \\
\hline Surgery before infection & 1.21 & $\begin{array}{l}0.98- \\
1.50\end{array}$ & 0.07 & & & \\
\hline $\begin{array}{l}\text { Neurosurgery within } 4 \text { weeks prior to } \\
\text { infections }\end{array}$ & 2.82 & $\begin{array}{l}1.56- \\
5.07\end{array}$ & $<0.001$ & 2.92 & $\begin{array}{l}1.48- \\
5.75\end{array}$ & 0.002 \\
\hline Immunosuppression state & 0.71 & $\begin{array}{l}0.52- \\
0.98\end{array}$ & 0.04 & & & \\
\hline Chronic kidney disease & 0.60 & $\begin{array}{l}0.33- \\
1.08\end{array}$ & 0.08 & & & \\
\hline Cerebral vascular disease & 1.65 & $\begin{array}{l}1.07- \\
2.53\end{array}$ & 0.02 & & & \\
\hline Liver abscess & 0.45 & $\begin{array}{l}0.04- \\
4.88\end{array}$ & 0.60 & & & \\
\hline Non-hepatic abscess & 1.19 & $\begin{array}{l}0.58- \\
2.44\end{array}$ & 0.64 & & & \\
\hline Previous exposure to cephalosporins & 2.90 & $\begin{array}{l}0.97- \\
8.72\end{array}$ & 0.045 & & & \\
\hline
\end{tabular}

$\mathrm{OR}$, odds ratio; $\mathrm{Cl}$, confidence interval.

Table 3. Clinical outcomes of patients infected with pVir+-KPC-kp and pVir-KPC-kp. 


\begin{tabular}{|lllll|}
\hline Variable & $\begin{array}{l}\text { All isolates } \\
(\mathbf{n = 3 5 2 )}\end{array}$ & $\begin{array}{l}\text { pVir-KPC-kp } \\
(\mathbf{n = 1 8 6 )}\end{array}$ & $\begin{array}{l}\text { pVir-KPC-kp } \\
(\mathbf{n = 1 6 6 )}\end{array}$ & $P$-value \\
\hline $\begin{array}{l}\text { Appropriate empirical } \\
\text { antimicrobial therapy }\end{array}$ & $58(16.5)$ & $37(19.9)$ & $21(12.7)$ & 0.07 \\
\hline $\begin{array}{l}\text { Appropriate definite antimicrobial } \\
\text { therapy }\end{array}$ & $205(58.2)$ & $116(62.4)$ & $89(53.6)$ & 0.10 \\
\hline MODS & $142(40.3)$ & $69(37.1)$ & $73(44.0)$ & 0.28 \\
\hline Septic shock & $93(26.4)$ & $58(31.2)$ & $35(21.1)$ & 0.03 \\
\hline $\begin{array}{l}\text { LOS from culture to discharge, } \\
\text { days, median (IQR) }\end{array}$ & $17(7-120)$ & $16(6.8-120)$ & $19(8-120)$ & 0.42 \\
\hline Mortality & $73(20.7)$ & $43(23.1)$ & $30(18.1)$ & 0.24 \\
\hline 7-day mortality & $158(44.9)$ & $85(45.7)$ & $73(44.0)$ & 0.75 \\
\hline 28-day mortality & $131(37.2)$ & $74(39.8)$ & $57(34.3)$ & 0.29 \\
\hline Infection-related mortality & $191(54.3)$ & $107(57.5)$ & $84(50.6)$ & 0.19 \\
\hline In-hospital mortality & & & & \\
\hline
\end{tabular}

Data are presented as No. (\%) unless otherwise specified;

MODS, multiple organ dysfunction syndrome; LOS, length of stay; IQR, interquartile range.

\section{Figures}




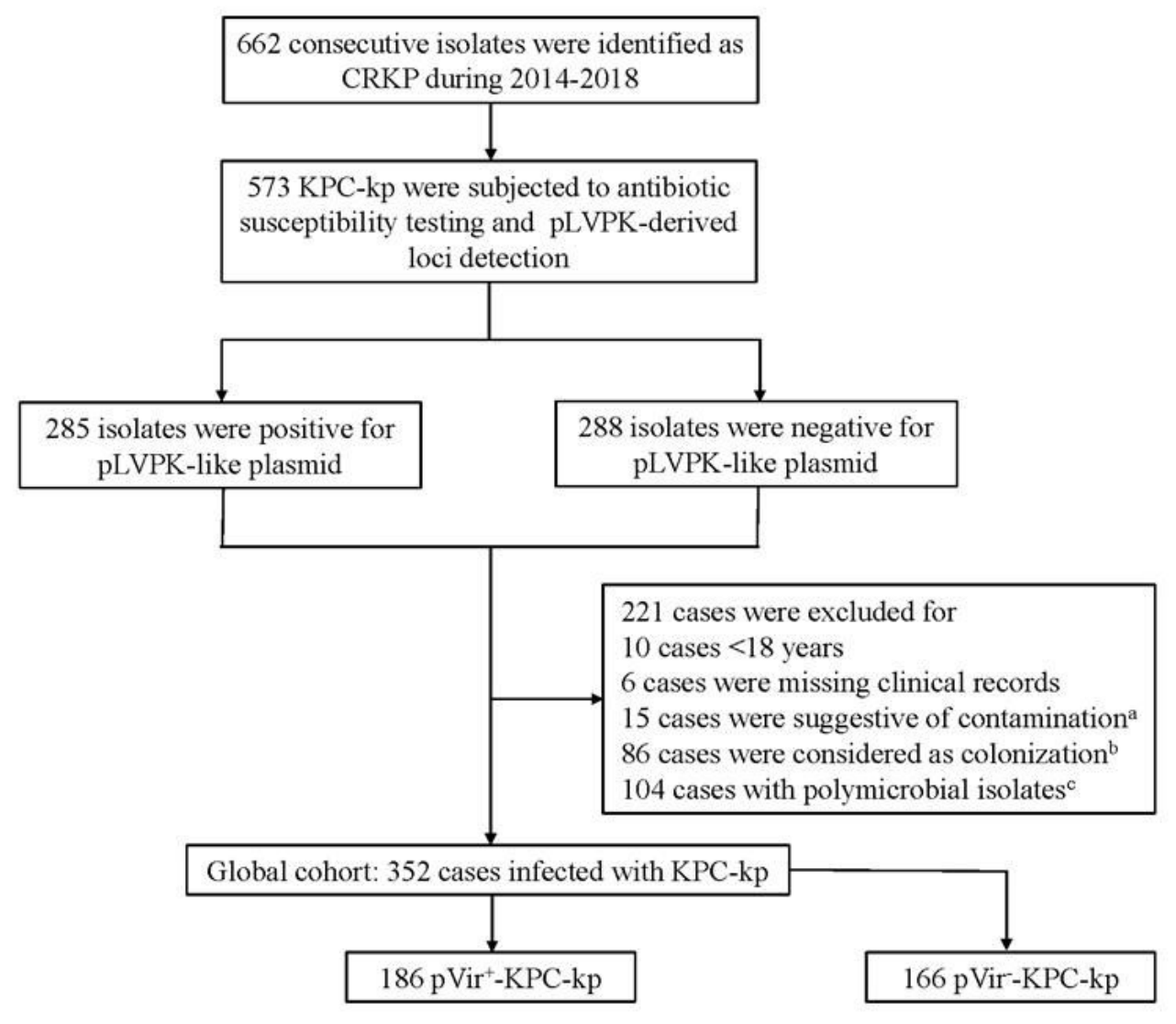

\section{Figure 1}

Flowchart for selection of 352 patients with KPC-kp infections. acontamination is defined as the presence of K. pneumonia in sterile fluid, without clinical signs or symptoms suggestive of infection; bcolonization is defines as the presence of $\mathrm{K}$. pneumonia in a sputum, stool or urine specimen, in the absence of systemic signs of inflammatory response; clncluding 41 case with Acinetobacter baumannii, 22 case with Pseudomonas aeruginosa, 11 case with Enterococcus faecium, 8 case with Stenotrophomonas maltophilia, 5 case with Escherichia coli, 3 case with Staphylococcus aureus, 3 case with Elizabethkingia meningoseptica, 2 case with Serratia marcescens, 2 case with Enterobacter cloacae, 1 case with Morganella morganii, 1 case with Burkholderia cepacia, 1 case with Enterococcus faecalis, 1 with Moraxella catarrhalis, 1 case with Cryptococcus neoformans, 1 case with Candida tropicalis and 1 case with Candida glabrata. 


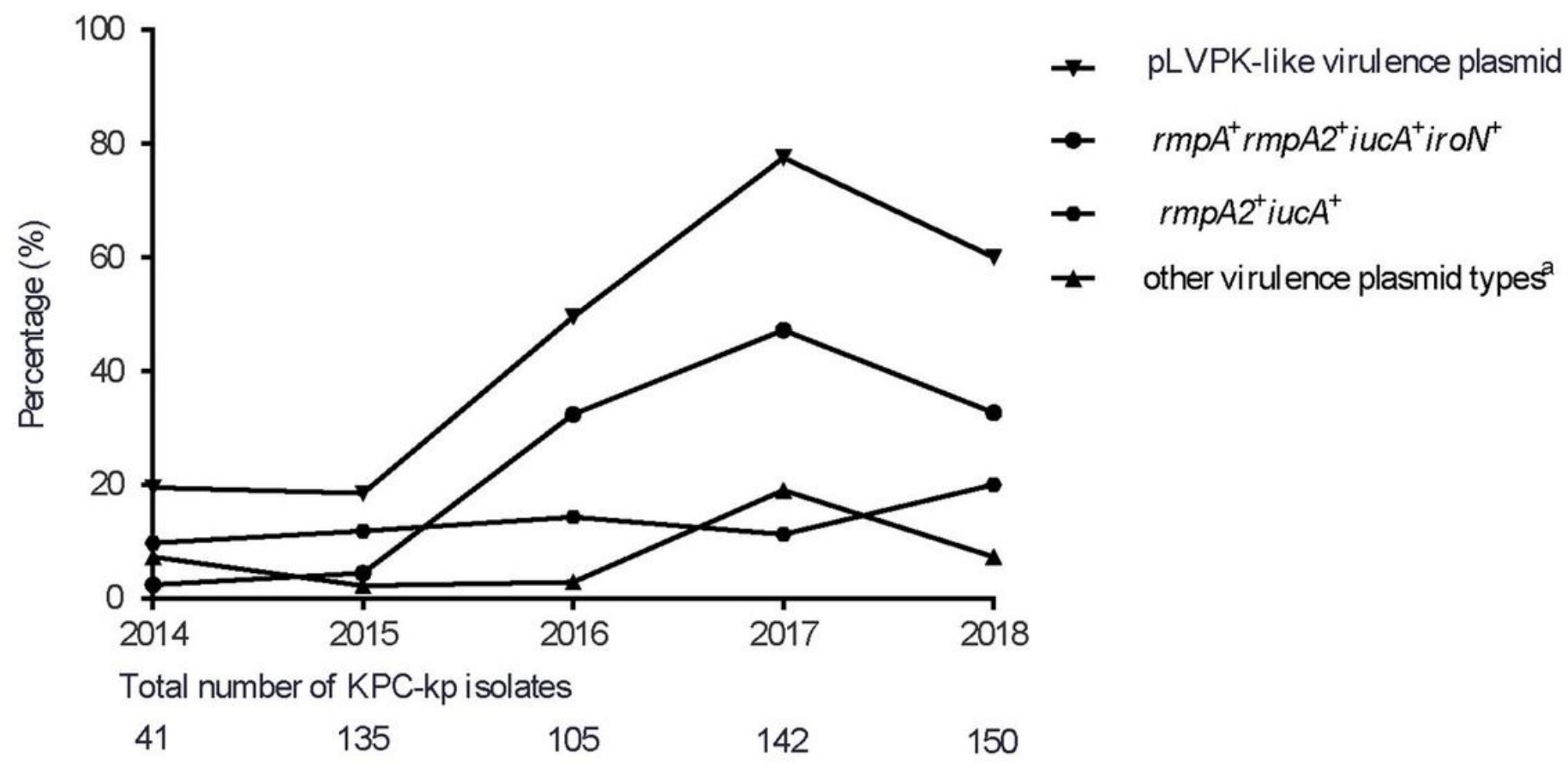

Figure 2

Annual distribution of the pVir types. a listed in Table S1. 


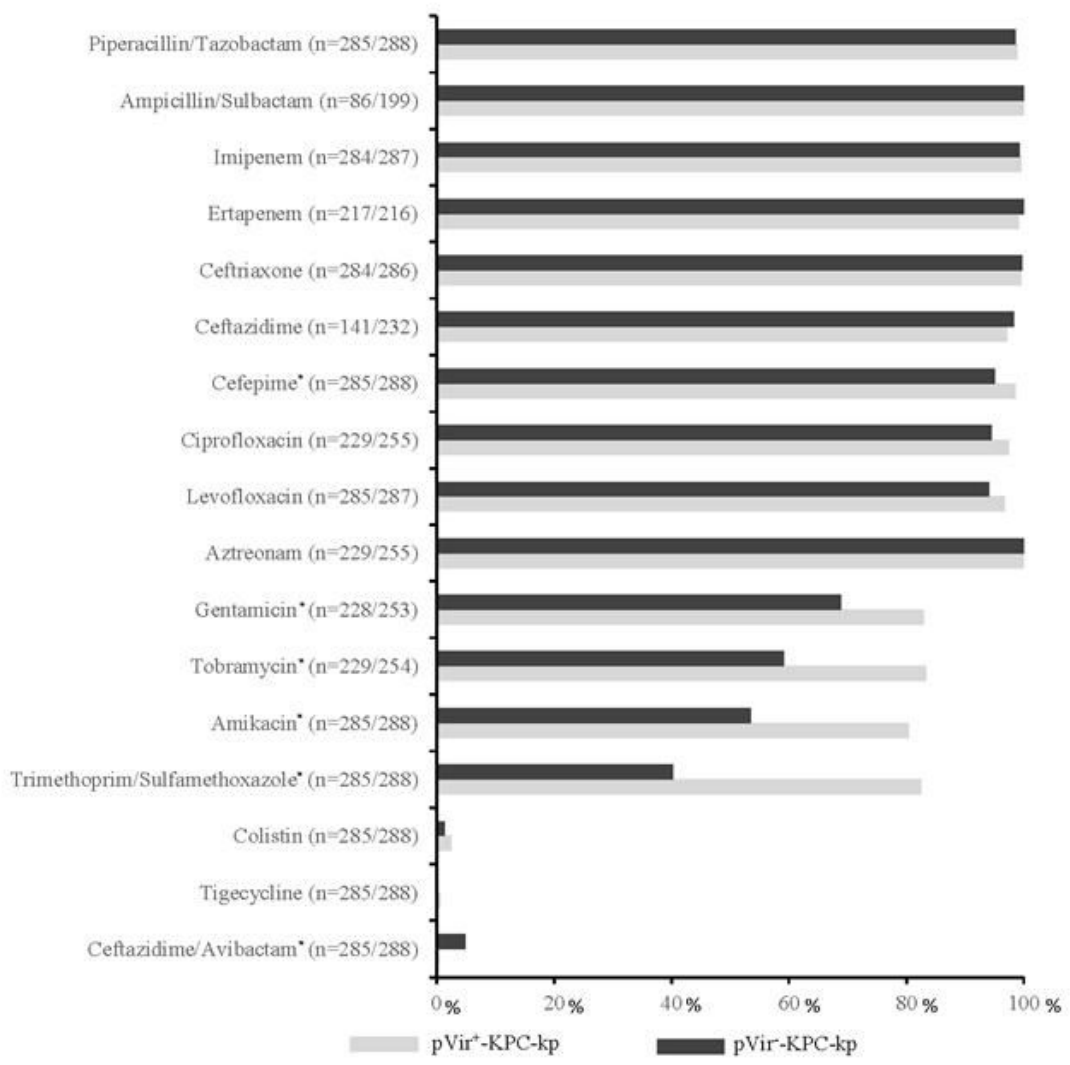

Figure 3

A comparison of the antibiotic susceptibility testing between pVir+-KPC-kp and pVir-KPC-kp strains. *P $<0.05$ 